\title{
MAGNETIC FIELD FLUCTUATIONS IN SC DIPOLE MAGNET
}

\author{
V. Shiltsev, FNAL, Batavia, IL 60510, USA \\ B.Baklakov, S.Singatulin, Budker INP, Novosibirsk, Russia 630090
}

\begin{abstract}
Magnetic field fluctuations at the betatron frequency can lead to emittance growth in circular accelerators. Tolerances are extremely tight for large hadron colliders like LHC and VLHC[1]. We performed experimental studies of the fluctuations in a stand-alone superconducting Tevatron magnet. Here we give a general description of the experimental set-up, present main results and discuss consequences for the colliders.
\end{abstract}

\section{INTRODUCTION}

Fig.1 shows general layout of the experimental set-up. Two 930 turn coils (dimensions $50 \mathrm{~cm} \times 2 \mathrm{~cm} \times 1 \mathrm{~cm}$ ) are set one after another on a G10 bar which is inserted inside the Tevatron dipole magnet. The magnet has been operated in a stand-alone mode in the magnet test facility (MTF) of the Fermilab Technical Division. It has a separate power supply to provide some 4T (vertical) dipole magnetic field at 4,000 A of current in superconducting coils. The plane of the measurement coils (see Fig.1) is perpendicular to the magnetic field, so any variation of the magnetic field flux through the coil results in induced voltage

$$
d U=-d(\text { Flux }) / d t=-2 \pi f d B * A
$$

where $f$ is frequency and $A=100 \mathrm{~cm}^{2}$ is the coil area. The induced voltage was measured by HP3458A digital voltmeter (DMM) with 19 bit resolution at $50 \mathrm{kHz}$ sampling rate.

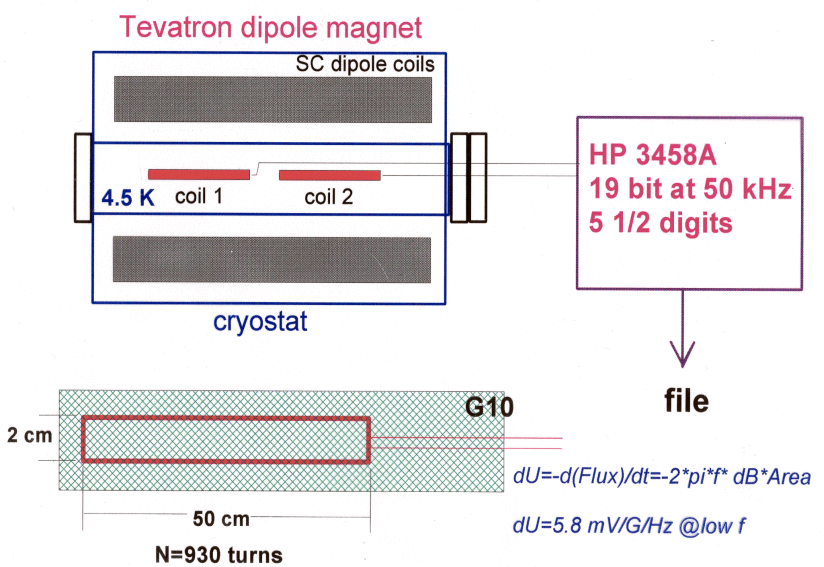

Figure 1: General layout of the set-up to measure $d B / B$ fluctuations.

Because of the coil capacitance and resistance, its frequency characteristics differs from pure inductance. Result of the coil sensitivity calibration is presented in Fig.2. One can clearly see a resonant peak with $Q \approx 20$ at $f=10 \mathrm{kHz}$. The calibration was performed at room temperature, while the coil stays at about $4.5^{\circ} \mathrm{K}$ when inside the SC Tevatron dipole. Because of the material shrinking, the resonant peak may be shifted at low temperatures as well as change its quality factor. We had no possibility to calibrate the coils at the liquid Helium temperatures.

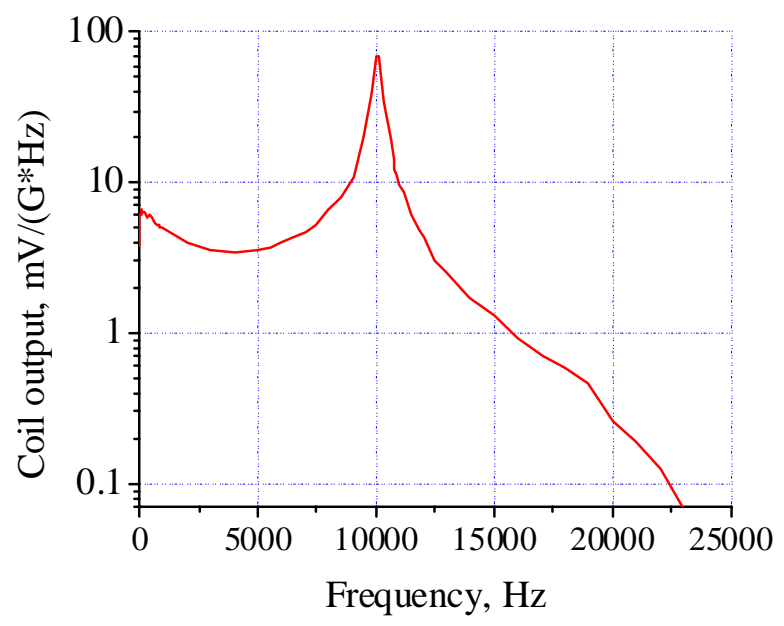

Figure 2: Sensitivity of the measurement coil.

\section{RESULTS}

The field fluctuation measurements were carried out in October 1999 at the Fermilab TD/MTF.

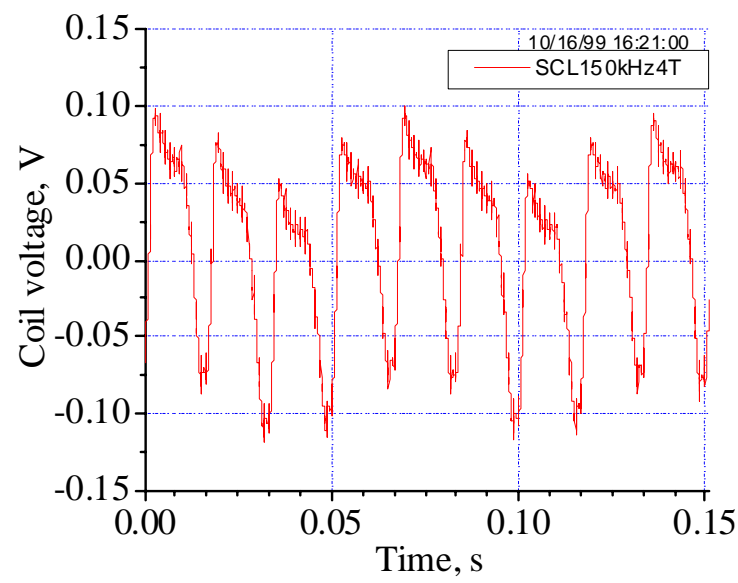

Figure 3: Coil voltage induced at 4T magnetic field.

Fig. 3 shows a typical time record of the coil voltage induced in $4 \mathrm{~T}$ field. The most visible is $60 \mathrm{~Hz}$ component, while high-frequency components are seen, too. The time records were processed by the FFT with Hanning window and resulting spectra are presented in Fig.4. Here the 
lowest curve shows the spectra of the DMM noise measured when the DMM input was connected to the ground. Two intermediate curves reflect noises in the coil with the magnet power supply off and with the power supply on but set at zero current. Finally, two upper curves are for the voltages induced in the coil at $2 \mathrm{~T}$ and $4 \mathrm{~T}$ magnetic field

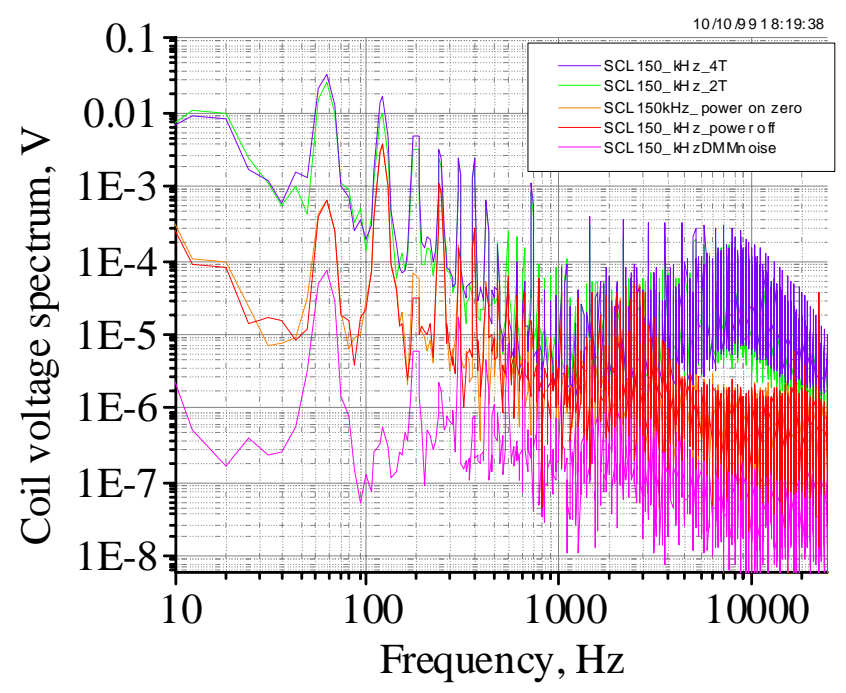

Figure 4: Coil voltage spectra.

One can conclude that the signal to noise ratio exceeds 10 over the entire frequency range of 10 to $25,000 \mathrm{~Hz}$.

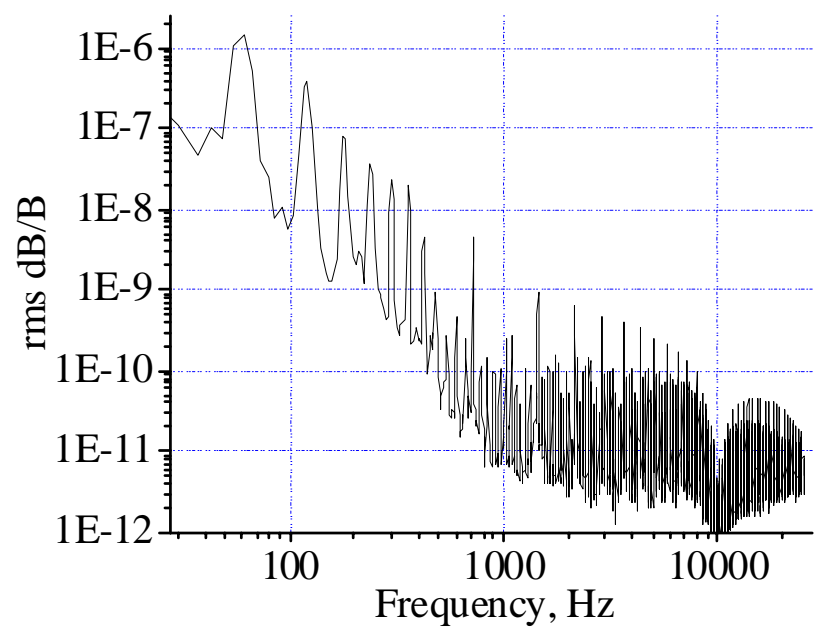

Figure 5: Rms field fluctuations in Tevatron dipole at 4T.

Applying calibration data to the raw signal spectrum and dividing by the maximum DC magnetic field we get rms $d B / B$ fluctuation spectrum as depicted in Fig.5. One can see following remarkable features: a) the maximum noise amplitude takes place at $60 \mathrm{~Hz}$ and does not exceed the value of $2 \times 10^{-6}$; b) $60 \mathrm{~Hz}$ harmonics dominate the spectrum until about $1000 \mathrm{~Hz}$, the harmonic amplitude goes down approximately as $1 / f^{3}$; c) there is a dip at $10,000 \mathrm{~Hz}$ that reflects known coil calibration which may be incorrect at LHe temperatures because we do not see any narrow peak at $10 \mathrm{kHz}$ in the raw data spectra - see Fig.4; d) above 700-1000 Hz, the spectrum flattens. The latter is of certain interest as that frequency range includes betatron frequencies of the VLHC, the LHC and the Tevatron.

We studied these excessive fluctuations in the second coil and found that while signals $U 1$ and $U 2$ look the same, the difference voltage between them grows at frequencies above $600 \mathrm{~Hz}$ - see Fig.6. The value of rms $(U 1-U 2)(f) \quad / r m s \quad U 1(f)$ is about 0.007-0.009 at frequencies below $400 \mathrm{~Hz}$, but it grows up to 0.3-0.5 above $600 \mathrm{~Hz}$.

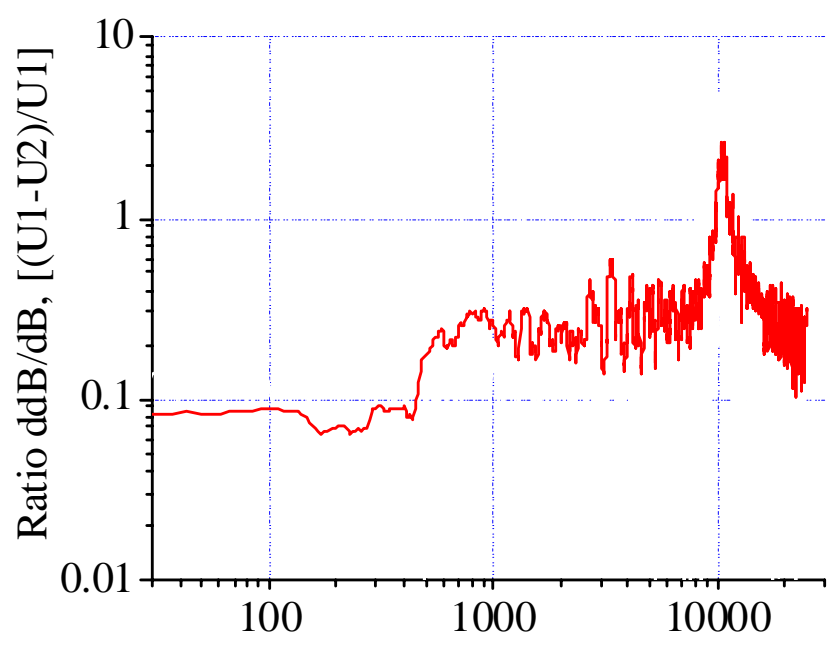

Frequency, $\mathrm{Hz}$

Figure 6: Spectral ratio of (signal difference)/(signal) .

One of the possible explanations of such behavior is that the field fluctuations are excited by sound waves due to turbulent flow of liquid Helium. The broad band turbulence leads to jitter of the beam pipe walls, the beam pipe changes its shape (quadrupole oscillation) that results in the magnetic field fluctuations because of the "frozen magnetic flux" effect at high frequencies [2]. Indeed, the beam pipe radius variation of $\delta R$ will result in the field variation of $\delta B / B=-\delta R / R$. For the Tevatron dipole pipe radius $R=35 \mathrm{~mm}$, and one needs only $\delta R=10^{-6} \mu \mathrm{m}$ to get the observed value of $\delta B / B=3 \times 10^{-11}$. Noteworthy to mention that $\delta R=10^{-4} \mu \mathrm{m}$ vibrations in the frequency range of $600-1400 \mathrm{~Hz}$ were observed on the cold mass of the SSC dipole magnet [3].

\section{EMITTANCE GROWTH DUE TO MAGNETIC FIELD FLUCTUATIONS}

Magnetic field fluctuations at the betatron frequency will cause miniscule turn-to-turn variation of the bending 
angle $\delta \theta=\theta_{0} \delta B / B$ in each dipole magnet and that will lead to the horizontal emittance growth [1] as:

$$
d \varepsilon_{N} / d t=f_{O} \gamma \beta_{\mathrm{ave}}\left(\delta B_{\mathrm{eff}} / B\right)^{2} /(2 N)
$$

where $f_{\mathrm{o}}$ is the revolution frequency, $\gamma$ is the relativistic factor, $\beta_{\text {ave }}$ is average beta-function, $N$ is the total number of dipoles and $\delta B_{\text {eff }} / B$ is the effective rms amplitude of the field fluctuations which for "colored" noise with power spectral density $S(f)$ can be defined as

$$
\left(\delta B_{\text {eff }} / B\right)=\left[2 f_{\mathrm{o}} \Sigma S\left(f_{\mathrm{o}}|n-Q|\right)\right]^{1 / 2}
$$

$Q$ is the horizontal tune. Table 1 presents relevant parameters of large hadron colliders and values of $\delta B_{\text {eff }} / B$ which will lead the emittance doubling over the characteristic time intervals (store time for VLHC-Stage I, LHC and Tevatron, synchrotron radiation damping time for VLHC-Stage II).

Table 1: Parameters and $\delta B_{\text {eff }} / B$ tolerances for large hadron colliders

\begin{tabular}{|lllll|}
\hline & VLHC-I & VLHC-II & LHC & Tevatron \\
$\mathcal{E}_{N}, \mu \mathrm{m}$ & 1.5 & 0.2 & 3.75 & 3.3 \\
$\tau, \mathrm{hrs}$ & 10 & 2 & 10 & 10 \\
$\varepsilon_{N} / \tau, \mathrm{fm} / \mathrm{s}$ & 40 & 27 & 100 & 90 \\
$\gamma$ & 20000 & 87000 & 7000 & 1000 \\
$f_{o}, \mathrm{kHz}$ & 1.3 & 1.3 & 11.3 & 48 \\
$N$ & 3440 & 13800 & 1200 & 774 \\
$\beta_{\text {ave }}, \mathrm{m}$ & 170 & 170 & 67 & 50 \\
$Q$ & 212.3 & 212.3 & 63.3 & 20.55 \\
& & & & \\
$f_{\text {res }}=f_{o} \Delta Q, \mathrm{~Hz}$ & 400 & 400 & 3400 & 20000 \\
$\delta B_{\text {eff }} / B, 10^{-10}$ & 7.8 & 6.0 & 2.8 & 2.1 \\
\hline
\end{tabular}

In Fig.7 we compare the tolerances with the equivalent field fluctuation amplitude calculated from experimental data for the 4T Tevatron magnet as $(\delta B / B)^{*}=[2 f \Sigma S(f)]^{1 / 2}$ -compare with Eq.(3). One can see that the tolerances are about equal or less than the experimental data. That situation requires following comments: first, contrary to Fig.6, there was no indication in the Tevatron that the beam emittance grows due to the external noises - most of the growth is due to intra-beam scattering (emittance doubles in some 10 hours). The contradiction can be explained as that each magnet of the machine is a part of a long magnet line which has in total very large inductance which allows to filter $60 \mathrm{~Hz}$ (power line) harmonic very effectively, especially at high frequencies. The second possible reason of the absence of the emitance growth is that the rms field fluctuations are measured over 0.5 long section of $6 \mathrm{~m}$ long magnet, and it seems quite possible that the magnetic field fluctuations in different parts of the magnet may cancel each other, thus, producing less effect.
Fig. 6 is quite in line with such a supposition. We also believe that the turbulent Helium effect should have coherence length much smaller than the magnet length (probably, comparable with the magnet transverse dimensions).

As for the other colliders, we have to note that the field fluctuations depend not only on the power supply stabilization but also on the magnet design. Therefore, no one expects to have the same fluctuations in the LHC and VLHC dipoles as in the Tevatron, because the magnets are very different from each other. Each of the magnet types needs to be measured separately for making specific predictions.

And finally, it is known that a low-noise feedback system which damps coherent beam oscillations caused by turn-by-turn noises can effectively reduce the emittance growth rate [4].

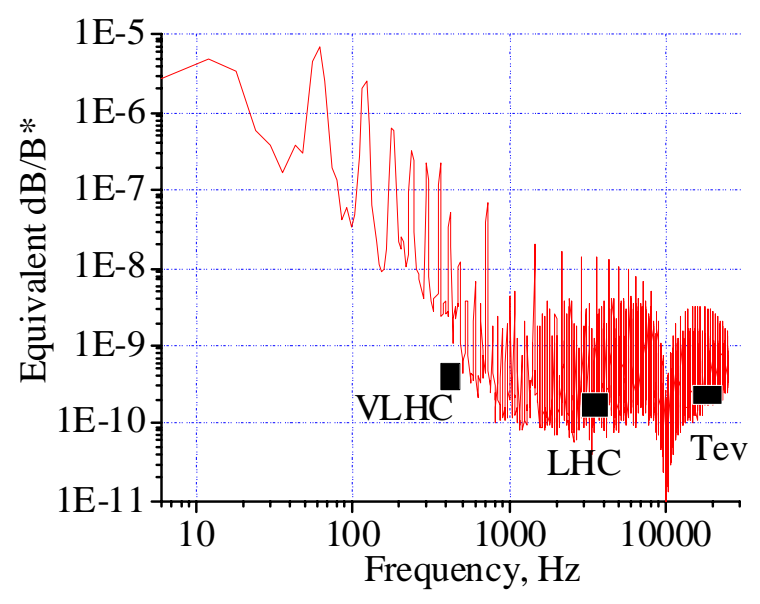

Figure 7: Equivalent rms field fluctuations in Tevatron dipole at $4 \mathrm{~T}$ and tolerances for large hadron colliders.

Our future plans include magnetic field fluctuation measurements in the low-field VLHC SC dipole magnet and in the LHC SC final focus quadrupole magnets, which are under construction at Fermilab. In the case of quadrupoles, a system of four orthogonal coils will be used.

We are thankful to P.Schlabach, M.Tartaglia, D.Orris and P.Aarseth for valuable assistance in our studies.

\section{REFERENCES}

[1] V.Lebedev, et.al, Part.accel, 44 (1994), 147.

[2] V.Parkhomchuk, V.Shiltsev, SSCL-622 (1993).

[3] V.Shiltsev et. al, AIP Conf.Proc., 326 (1995), 577.

[4] A.Burov, et.al, NIM-A, 450 (2000), 194. 